\title{
Efecto del Extracto de Maíz Morado "Chicha Morada" durante el blanqueamiento Dental. In vitro
}

\author{
Effect of Purple Corn Beverage "Chicha Morada" \\ During Dental Bleaching. In vitro
}

\author{
Katherine Vilchez Fuentes Rivera'; Francisco Aurelio Rumiche² \& Lidia Yileng Tay ${ }^{1}$
}

VILCHEZ, F. R. K.; RUMICHE, F. A. \& TAY, L. Y. Efecto del extracto de maíz morado "Chicha Morada" durante el blanqueamiento dental. In vitro. Int. J. Odontostomat., 12(4):416-422, 2018.

RESUMEN: El resultado del tratamiento de blanqueamiento dental puede verse afectado en pacientes que consumen bebidas pigmentantes durante el tratamiento. El objetivo de este trabajo consistió en valuar el efecto in vitro de la exposición al extracto de maíz morado (chicha morada) sobre el color del esmalte humano, durante y después del tratamiento del blanqueamiento dental con peróxido de hidrógeno al 35 \%. Se utilizaron 48 dientes humanos, divididos en grupos según la bebida a la que se expuso: Extracto de maíz morado peruano (MM), té verde (T) y agua destilada $(A)$; la mitad de los especímenes expuestos a cada bebida fueron sometidos a blanqueamiento dental con peróxido de hidrógeno al $35 \%$ durante los primeros días de exposición a la pigmentación, resultando en los siguientes grupos: Grupo A: Sin blanqueamiento + maíz morado, Grupo B: Sin blanqueamiento + té verde, Grupo C: Sin blanqueamiento + agua destilada, Grupo D: Con blanqueamiento + maíz morado, Grupo E: Con blanqueamiento + té verde, Grupo F: Con blanqueamiento + agua destilada. Los cambios de color se midieron con un espectrofotómetro digital (VITA Easyshade Advance 4.0, VITA, Alemania) antes del blanqueamiento, durante el blanqueamiento, finalizado el blanqueamiento y al final de los 36 días de exposición a los pigmentos. Según el $\Delta \mathrm{E}$, el extracto de maíz morado difiere significativamente con el agua destilada ( $p<$ $0,05)$. Con respecto al grado de luminosidad, el extracto de maíz morado con blanqueamiento presentó los menores valores de luminosidad $(p<0,05)$. En cuanto al croma, no hubo diferencias entre los grupos $(p>0,05)$. La exposición al extracto de maíz morado pigmenta los dientes, durante el blanqueamiento el extracto de maíz morado no afecta el tratamiento, pero si la exposición continúa luego del blanqueamiento dental, el color de los dientes se verá afectado.

PALABRAS CLAVE: peróxido de hidrógeno, blanqueamiento de dientes, pigmentación.

\section{INTRODUCCIÓN}

Hoy en día es común encontrar pacientes que exigen estética en la consulta odontológica, siendo que la mayoría de estos pacientes solicitan blanqueamiento dental por insatisfacción con el color de sus dientes. Para tratar la alteración de color existen diversos tratamientos, los más invasivos son los restauradores ya sea con carillas de resina o carillas de cerámica y el menos invasivo es el blanqueamiento dental, siendo este último una alternativa conservadora y exitosa para conseguir la satisfacción de estos pacientes (Pirolo et al., 2014).

Se ha reportado que ciertas sustancias, principalmente alimentos y bebidas oscuras como el café, vino tinto, té, gaseosa y bebidas energizantes pigmentan los dientes (Pirolo et al.; Matis et al., 2015; Briso et al., 2016). Estos cambios pueden estar asociados a pigmentos que se adhieren en la superficie dental (Azer et al., 2011) pero diversos autores refieren que el cambio de color de los dientes va a depender de diferentes factores, unos propios de la solución pigmentante (Berger et al., 2008; Attia et al., 2009; Azer et al.; Erdemir et al., 2012; Côrtes et al., 2013; Karadas \& Seven, 2014) y otros dependiendo de la propia estructura dental. Luego del blanqueamiento dental se obtienen ligeras alteraciones morfológicas en la superficie dental; la composición del esmalte, la absorción de agua, alteraciones en la permeabilidad e irregu-

\footnotetext{
${ }^{1}$ Odontología Restauradora y Estética. Facultad de Estomatología. Universidad Peruana Cayetano Heredia, Perú.

${ }^{2}$ Ingeniería de Materiales. Facultad de Ingeniería Mecánica. Pontificia Universidad Católica del Perú, Perú. 
laridades que pueden quedar luego del blanqueamiento, podrían facilitar la acumulación de pigmentos (Dahl \& Pallesen, 2003). Estos cambios en los dientes no son perceptibles por el ojo humano (Ruyter et al., 1987).

Una de las bebidas más consumidas en el Perú es la "chicha morada", la cual tiene una coloración oscura. A pesar de ser una bebida oscura, hay pocos estudios que demuestran si esto puede afectar el blanqueamiento dental (Castillo-Ghiotto et al., 2013). El principal ingrediente de la chicha morada es el maíz morado, éste tiene un pigmento llamado antocianina, el cual le da el color característico y debe ser el responsable de pigmentar los dientes durante la exposición (Ramos-Escudero et al., 2012). Otros alimentos que contienen dicho pigmento son los arándanos, uvas rojas, vino tinto y acai (de Alencar E Silva Leite et al., 2014). Las antocianinas son pigmentos vacuolares solubles en agua que pueden aparecer rojos, morados o azules, de acuerdo al $\mathrm{pH}$ (Passamonti et al., 2005; da Costa e Silva et al., 2009; de Alencar E Silva Leite et al.).

El objetivo del presente estudio fue evaluar in vitro el efecto de la exposición de los dientes al extracto de maíz morado "chicha morada" sobre el color del esmalte humano, durante y después del tratamiento de blanqueamiento dental.

\section{MATERIAL Y MÉTODO}

Obtención de los especímenes. Este estudio fue aprobado por el Comité Institucional de Ética (CIE) de la Universidad Peruana Cayetano Heredia (227-13-15).

Para el estudio se utilizaron 48 dientes extraídos por motivos ortodónticos. Los especímenes fueron divididos en 6 grupos y fueron expuestos a diferentes sustancias durante 36 días seguidos. La mitad de estos especímenes fueron expuestos a blanqueamiento dental. Los grupos resultantes se describen de la siguiente manera:

\footnotetext{
Grupo A $(n=8)$ : Sin blanqueamiento + maíz morado (SBMM) (SBT)

Grupo B $(n=8)$ : Sin blanqueamiento + té verde

. Grupo $C(n=8)$ : Sin blanqueamiento + agua destilada (SBA)

- Grupo $D(n=8)$ : Con blanqueamiento + maíz morado (BMM)
}

Grupo $E(n=8)$ : Con blanqueamiento + té verde

$$
\text { - Grupo } F(n=8) \text { : Con blanqueamiento + agua desti- }
$$
lada (BA)

Los criterios para incluir los dientes humanos al estudio para la preparación de los especímenes de esmalte fueron: Dientes libres de lesiones cariosas y no cariosas, dientes de reciente extracción, dientes sin grietas, dientes sin fracturas, y dientes sin alteraciones en la superficie del esmalte.

Para obtener los especímenes, los dientes fueron lavados con agua destilada y seccionados con un disco diamantado (KG Sorensen $\AA$, Brasil) bajo refrigeración constante con agua. Primero se separó la corona de la raíz con un corte a nivel amelo-cementario y posteriormente se realizó un corte en sentido mesiodistal para la obtención de dos fragmentos dentarios.

Estos fragmentos fueron embebidos en un tubo de PVC de $10 \mathrm{~mm}$ de diámetro con resina acrílica Vitacryl (Vitalloy, Perú), dejando la superficie del esmalte expuesta.

Los especímenes fueron sometidos a un aplanamiento superficial del esmalte con lijas de agua de granos 400, 600 y 800 (ASAlite, Perú) durante 30 segundos y para pulir la superficie se utilizaron lijas de granos 1000, 1500 y 2000 (ASAlite, Perú) durante 60 segundos con agua, verificando el pulido obtenido antes de pasar a la siguiente más fina. Además los especímenes fueron lavados antes de pasar a la lija de la siguiente granulación (una lija nueva para cada pulido).

Finalmente fueron almacenados en agua destilada.

Luego, todos los especímenes se colocaron en té negro (Hornimans, España) durante 72 horas, para simular situaciones clínicas donde haya necesidad de tratamiento de blanqueamiento.

1. Preparación de las sustancias pigmentantes. Extracto de maíz morado: $1 \mathrm{~kg}$ de maíz morado hervido en un litro de agua.

Té verde: Fueron utilizadas cinco bolsitas filtrantes de té (Lipton, Chile) por litro de agua.

Para ambos casos la preparación se realizó dejando hervir agua con la sustancia a utilizar por 15 minutos a temperatura de ebullición. Finalmente se dejaron enfriar hasta temperatura ambiente. 
2. Exposición a sustancias pigmentantes. Los especímenes se sumergieron en $20 \mathrm{ml}$ de la sustancia pigmentante correspondiente a su grupo (Extracto de maíz morado, té verde o agua destilada) en un vaso de plástico 30 minutos diarios por 36 días a temperatura ambiente. Luego de la exposición a sustancias pigmentantes se lavaron los especímenes con agua destilada y se almacenaron nuevamente en agua destilada en recipientes de plástico rotulados, hasta que los procedimientos del experimento se llevaran a cabo.

3. Procedimiento de blanqueamiento. El tratamiento de blanqueamiento se realizó de acuerdo con procedimientos estándar.

El peróxido de hidrógeno al 35 \% (HP Maxx, FGM, Brazil) fue colocado sobre la superficie de los especímenes, siguiendo las instrucciones del fabricante, en dos aplicaciones de 15 minutos, y esto fue repetido en una segunda sesión después de una semana.

4. Registro del color. Para realizar la toma de color de los especímenes, se utilizó un espectrofotómetro digital (VITA Easyshade Advance 4.0, VITA, Alemania).

La punta lectora del espectrofotómetro digital fue colocada directamente sobre el espécimen con la ayuda de la matriz de silicona como guía.

Fueron realizadas en un mismo ambiente a la misma hora del día para estandarizar los especímenes.

Después del disparo de la luz fueron registrados los valores de la escala VITA y 3D MASTER, DE (diferencia de color), $L^{*}$ (luminosidad), $a^{*}\left(-a^{*}=\right.$ verde; $+a^{*}=$ rojo), b* (-b* = azul; +b* = amarillo), h (matiz) y c (croma) de cada espécimen, obteniendo de esa forma los valores iniciales de color.

Se tomaron tres registros de color por espécimen. Las tomas de color se realizaron en los siguientes tiempos: T0 - Al inicio de los procedimientos, T1 - Después de la primera aplicación de blanqueamiento, T2 - Antes de la segunda aplicación de blanqueamiento, T3 - Después de la segunda aplicación de blanqueamiento, T4 - A la semana después de la segunda aplicación, T5 - A las 2 semanas después de la segunda aplicación, T6 - Al mes después de la segunda aplicación.
5. Plan de análisis. Para el análisis estadístico, se utilizaron 2 tiempos: Diferencia de T0-T3 (cambio de color durante el blanqueamiento) y diferencia de T0-T6 (cambio de color durante y después del blanqueamiento).

Los datos se analizaron con el paquete estadístico BioStat v5 para Windows.

Los datos presentaron distribución normal, por lo que se utilizó la prueba de ANOVA y el post test de Tukey $(p<0,05)$.

\section{RESULTADOS}

La Tabla I muestra los cambios de color en los grupos sin blanqueamiento y con blanqueamiento en diferentes tiempos: T0-T3, T0-T6. La Tabla II muestra el grado de luminosidad en los grupos sin blanqueamiento y con blanqueamiento en diferentes tiempos: T0-T3, T0-T6. La Tabla III muestra el grado de intensidad o croma en los grupos sin blanqueamiento y con blanqueamiento en diferentes tiempos: T0-T3, T0-T6.

Tabla I. Diferencia de $\Delta \mathrm{E}$ en los tiempos T0-T3 y T0-T6 de todos los grupos.

\begin{tabular}{ccc}
\hline Grupos & T0-T3 & T0-T6 \\
\hline A & $10,15(4,61) \mathrm{ab}$ & $9,41(3,8) \mathrm{a}$ \\
B & $7,26(4,53) \mathrm{ab}$ & $5,12(2,78) \mathrm{b}$ \\
C & $10,67(3,97) \mathrm{a}$ & $10,9(5,84) \mathrm{a}$ \\
D & $6,92(4,67) \mathrm{b}$ & $9,65(3,78) \mathrm{a}$ \\
E & $3,75(3,93) \mathrm{bc}$ & $12,05(2,99) \mathrm{a}$ \\
F & $3,29(1,79) \mathrm{c}$ & $9,39(5,31) \mathrm{a}$ \\
\hline
\end{tabular}

Letras diferentes en las columnas muestran diferencias estadísticamente significativas $(p<0,05)$ entre los grupos.

Tabla II. Diferencia de L en los tiempos T0-T3 y T0-T6 de todos los grupos.

\begin{tabular}{ccc}
\hline Grupos & T0-T3 & T0-T6 \\
\hline A & $10,45(3,02) \mathrm{e}$ & $21,97(3,50) \mathrm{a}$ \\
B & $5,1(4,76) \mathrm{a}$ & $9,67(5,94) \mathrm{b}$ \\
C & $2,01(3,45) \mathrm{ab}$ & $7,4(3,36) \mathrm{b}$ \\
D & $-6,29(3,71) \mathrm{c}$ & $18,77(4,23) \mathrm{a}$ \\
E & $-6,25(8,83) \mathrm{c}$ & $5,96(5,58) \mathrm{bc}$ \\
F & $-1,38(6,33) \mathrm{b}$ & $2,79(5,13) \mathrm{c}$ \\
\hline
\end{tabular}

Letras diferentes en las columnas muestran diferencias estadísticamente significativas $(p<0,05)$ entre los grupos. 
Tabla III. Diferencia de C en los tiempos T0-T3 y T0-T6 de todos los grupos.

\begin{tabular}{ccc}
\hline Grupos & T0-T3 & T0-T6 \\
\hline A & $9,50(6,56) \mathrm{ab}$ & $10,04(6,89) \mathrm{a}$ \\
B & $2,55(11,06) \mathrm{b}$ & $0,13(10,12) \mathrm{b}$ \\
C & $11,91(7,10) \mathrm{a}$ & $11,27(6,70) \mathrm{a}$ \\
D & $11,02(10,2) \mathrm{a}$ & $17,18(5,94) \mathrm{a}$ \\
E & $13,53(12,1) \mathrm{a}$ & $11,75(8,25) \mathrm{a}$ \\
F & $6,96(4,37) \mathrm{ab}$ & $13,96(3,65) \mathrm{a}$ \\
\hline
\end{tabular}

Letras diferentes en las columnas muestran diferencias estadísticamente significativas $(p<0,05)$ entre los grupos.

Tabla IV. Diferencia de h en los tiempos T0-T3 y T0-T6 de todos los grupos.

\begin{tabular}{ccc}
\hline Grupos & T0-T3 & T0-T6 \\
\hline A & $2,61(2,00) \mathrm{a}$ & $12,67(4,33) \mathrm{a}$ \\
B & $0,89(4,01) \mathrm{ab}$ & $3,62(2,93) \mathrm{b}$ \\
C & $-0,13(2,24) \mathrm{ab}$ & $0,604(1,77) \mathrm{bc}$ \\
D & $-2,67(4,49) \mathrm{b}$ & $14,208(5,16) \mathrm{a}$ \\
E & $-0,61(4,79) \mathrm{ab}$ & $-2,46(4,106) \mathrm{cd}$ \\
F & $-2,18(3,96) \mathrm{b}$ & $-5,51(3,35) \mathrm{d}$ \\
\hline
\end{tabular}

Letras diferentes en las columnas muestran diferencias estadísticamente significativas $(p<0,05)$ entre los grupos.

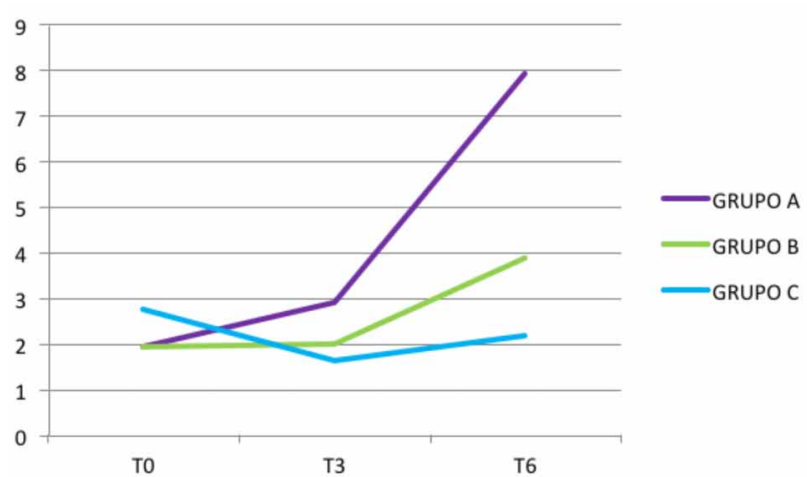

Fig. 1. Tendencia de $a^{*}$ según la escala $\mathrm{CIE} \mathrm{L}^{*} \mathrm{a}^{*} \mathrm{~b}^{*}$ en los tiempos T0, T3 y T6 de los grupos A, B y C.
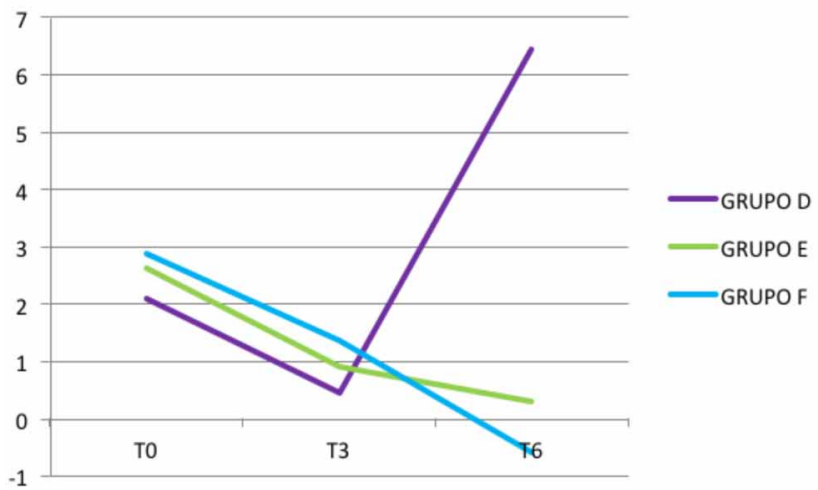

Fig. 2. Tendencia de $a^{*}$ según la escala CIE $L^{*} a^{*} b^{*}$ en los tiempos T0, T3 y T6 de los grupos D, E y F.
La Tabla IV muestra el grado de tonalidad en los grupos sin blanqueamiento y con blanqueamiento en diferentes tiempos: T0-T3, T0-T6.

La Figura 1 nos muestra que el extracto de maíz morado tuvo una tendencia hacia rojo.

La Figura 2 muestra que en la primera semana de blanqueamiento todos los grupos fueron yendo hacia 0 , eso significa que están eliminando los pigmentos rojos que antes tenían. Después, el extracto de maíz morado se volvió a pigmentar.

En la Figura 3 no hubo diferencia del extracto de maíz morado con los otros grupos porque los dientes tienen un tono amarillo, es por ese motivo que el número está alto.

En la Figura 4, el extracto de maíz morado tuvo una tendencia hacia el azul, en cambio el té verde mantuvo su color amarillo.

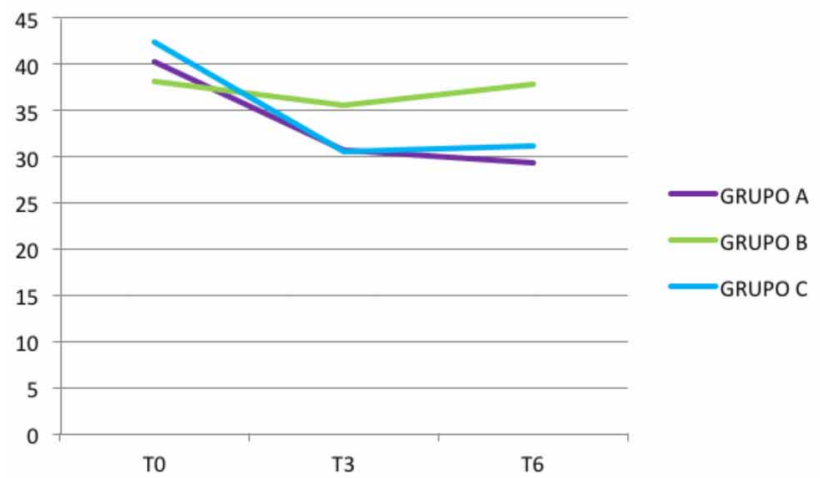

Fig. 3. Tendencia de $b^{*}$ según la escala CIE $L^{*} a^{*} b^{*}$ en los tiempos T0, T3 y T6 de los grupos A, B y C.

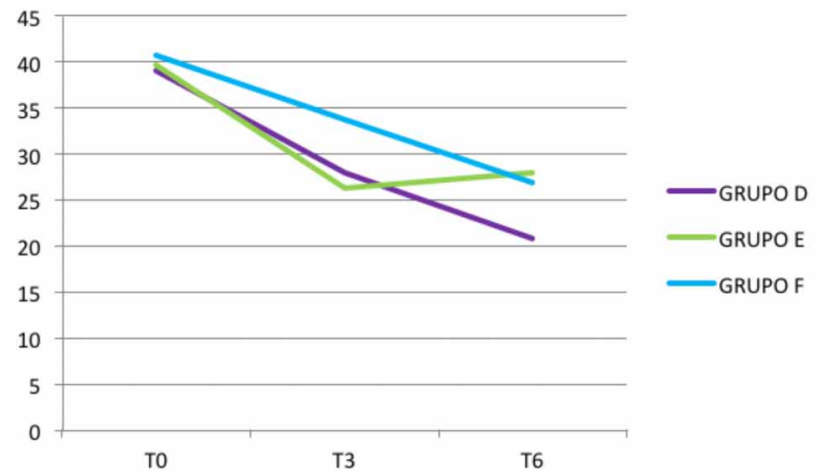

Fig. 4. Tendencia de $b^{*}$ según la escala CIE L*a*b* en los tiempos T0, T3 y T6 de los grupos D, E y F. 


\section{DISCUSIÓN}

El objetivo de este estudio fue evaluar el efecto in vitro de la exposición al extracto de maíz morado sobre el color del esmalte humano durante y después del blanqueamiento dental con peróxido de hidrógeno al $35 \%$. Encontramos que la exposición al extracto de maíz morado pigmenta los dientes; si la exposición es durante el blanqueamiento, ésta no afecta el resultado final de este tratamiento, sin embargo, si la exposición del extracto de maíz morado continúa luego del blanqueamiento dental, el color de los dientes se verá afectado.

La chicha morada tiene como principal ingrediente al maíz morado, éste tiene un pigmento llamado antocianina, el cual le da el color característico y debe ser el responsable de pigmentar los dientes durante la exposición (Ramos-Escudero et al.). Otros alimentos que contienen dicho pigmento son los arándanos, uvas rojas, vino tinto y acai (de Alencar E Silva Leite et al.). Las antocianinas son pigmentos vacuolares solubles en agua que pueden aparecer rojos, morados o azules, de acuerdo al pH (Passamonti et al.; da Costa e Silva et al.; de Alencar E Silva Leite et al.).

En base a nuestros conocimientos, el estudio se centró en investigar el efecto de exponer los dientes a chicha morada ya que es muy limitado. Castillo-Ghiotto et al. también demostraron que la chicha morada pigmenta dientes bovinos después de la exposición de 35 días.

Por otro lado, Acuña et al. (2016) llegaron a la conclusión de que el extracto de maíz morado, té verde y agua destilada generan cambios de color en la resina utilizada en su estudio. La exposición al extracto de maíz morado fue la única bebida que generó pigmentaciones perceptibles al ojo humano en discos de resina sin blanqueamiento. La exposición al extracto de maíz morado durante el procedimiento de blanqueamiento con peróxido de hidrógeno al 35 \% genera cambios de color, pero este cambio no es perceptible al ojo humano (Acuña et al.).

Otra bebida que se contraindica durante el blanqueamiento y que también contiene antocianina es el vino tinto. Berger et al. no recomiendan que los pacientes beban vino tinto durante el tratamiento de blanqueamiento porque durante este periodo el esmalte se encuentra más propenso a pigmentaciones por esta bebida (Berger et al.). Otro estudio presentado por Côrtes et al. concluyeron que los pacientes deben evitar el consumo de vino tinto después del tratamiento de blanqueamiento dental. de Araújo et al. (2013) evaluaron la estabilidad de color en el esmalte dental sometido a blanqueamiento con peróxido de carbamida al $10 \%$ combinado con soluciones pigmentantes (gaseosa, chocolate derretido y vino tinto), encontrando que el esmalte expuesto al vino tinto mostró la menor estabilidad de color. El vino tinto pigmenta por el pH ácido de la sustancia, por su contenido de etanol y a los pigmentos presentes en su composición (Berger et al.; Attia et al.; Côrtes et al.). Todos estos estudios demuestran que las bebidas a base de antocianinas son altamente pigmentantes para los dientes.

En el presente estudio se ha demostrado que si se expone el extracto de maíz morado durante el blanqueamiento, ésta no influye en el resultado inmediato de dicho tratamiento. Esto concuerda con otros estudios que han evaluado otras bebidas pigmentantes como el café, té y gaseosas oscuras. Algunos autores (Attia et al.; Liporoni et al., 2010; Bazzi et al., 2012) encontraron que la exposición al café no influyó en el resultado inmediato del blanqueamiento dental en estudios in vitro. Sin embargo, en la evaluación post-blanqueamiento se encontró que los especímenes fueron más inestables en el color, así como en el presente estudio. Lo mismo sucedió con el estudio de Côrtes et al., en la cual se permitió que los pacientes tomaran café durante el tratamiento de blanqueamiento sin afectar los resultados, sin embargo, si continúan con esos hábitos presentarán variaciones en la estabilidad de color. Estos resultados también fueron encontrados en un estudio realizado por Rezende et al. (2013), quienes concluyeron que el consumo de café durante el blanqueamiento no influye en el resultado final del tratamiento.

Con respecto al DE, según Ruyter et al., los cambios de $\Delta E$ menores a 3,3 no son perceptibles al ojo humano. En el presente estudio, los dientes expuestos al extracto de maíz morado tuvieron una diferencia de $\Delta \mathrm{E}$ mayor a 5 , siendo este cambio perceptible al ojo humano, además con los otros grupos se encontró diferencias de $\Delta E$ también mayores a 3,3; sin embargo, esto se puede deber a los cambios originados al retirar los pigmentos iniciales del té negro.

Si evaluamos por separado las coordenadas del espacio cromático de la escala CIELab, encontramos que en cuanto a la luminosidad, el extracto de maíz morado mostró mayor variación, siendo que el diente se oscurece más, a diferencia de la exposición a té verde 
y agua, lo cual corrobora que el pigmento del maíz morado, antocianina, hace que el diente se vea más oscuro. Además observamos que así como con el agua, los especímenes de té verde no variaron en cuanto a luminosidad, a pesar de que se ha demostrado que el té verde oscurece los dientes (Griffiths et al., 2008; Adeyemi et al., 2010; D'Arce et al., 2013), en este estudio no fue así, y puede deberse a la metodología utilizada, ya que los especímenes fueron pigmentados en un principio con té negro para homogenizar todas los especímenes, haciendo que el color inicial y el final después de la exposición a té no varíe.

Al analizar los especímenes durante el blanqueamiento, se muestra que los dientes de los tres grupos aclararon, siendo que los grupos de extracto de maíz morado y té verde aclararon más que el grupo que fue expuesto al agua. Esto puede deberse a que el extracto de maíz morado ayudaría en la penetración de los radicales libres de peróxido de hidrógeno a través del esmalte y la dentina permitiendo una mayor eliminación de pigmentos.

Sin embargo, una vez terminada la exposición al agente blanqueador, los especímenes expuestos al extracto de maíz morado se oscurecieron significativamente. Los especímenes expuestos a té verde y agua no mostraron variación significativa después del blanqueamiento. Hay una observación en la metodología utilizada, al tener desde un inicio los dientes pigmentados con té negro para homogenizar los especímenes, el grupo de té verde sólo mantuvo el mismo color inicial, hubo cambios durante el blanqueamiento, pero el resultado final fue el mismo que el color inicial. Esto podría dar la falsa impresión de que el té no pigmenta, cuando lo que sucedió es que se eliminaron los pigmentos en un inicio y luego el diente se repigmentó.

En cuanto al croma, se encontró que los grupos expuestos a extracto de maíz morado y agua destilada disminuyeron significativamente la saturación de color sin diferencias entre ellos, a diferencia del grupo expuesto a té verde en donde la variación de la saturación fue mínima, esto puede deberse a que el té verde mantiene la pigmentación del té negro.

Durante el blanqueamiento, el grupo con agua destilada mostró menores cambios de saturación a comparación del grupo de extracto de maíz morado y té verde. Así como en los resultados de luminosidad, esto puede deberse a que se eliminan los pigmentos iniciales de té negro, marcando la disminución en la satura- ción del color. En el grupo de agua destilada no hay cambios de saturación porque ésta no presenta pigmentos, a diferencia del extracto de maíz morado y el té verde, donde la saturación es la misma ya que se sigue saturando de pigmentos.

Sin embargo, en la evaluación completa con blanqueamiento y una semana después los tres grupos disminuyeron su saturación sin diferencias significativas entre ellos. Demostrando con estos resultados que el blanqueamiento dental disminuye el croma de los especímenes, es decir, todas los especímenes disminuyeron la saturación de color, haciendo que los dientes se vean más claros.

Cuando se evaluó el matiz o tonalidad, es decir el color del diente, se apreció que en el primer tiempo no hubieron diferencias estadísticamente significativas entre los grupos, sin embargo, a las 4 semanas hubo un cambio de color estadísticamente significativo para el extracto de maíz morado, es decir, el extracto de maíz morado cambia la tonalidad del diente. Cuando se evaluó el a y el b de la escala CIELab se encontró que los dientes que recibieron extracto de maíz morado tienden hacia el color rojo en la escala (a) y azul en la escala (b), coincidentemente con los colores que forman el color morado.

CONCLUSIONES. Dentro de las limitaciones de este estudio in vitro, La exposición al extracto de maíz morado pigmenta los dientes; si la exposición es durante el blanqueamiento, ésta no afecta el resultado final de este tratamiento, sin embargo, si la exposición al extracto de maíz morado continúa luego del blanqueamiento dental, el color de los dientes se verá afectado.

VILCHEZ, F. R. K.; RUMICHE, F. A. \& TAY, L. Y. Effect of purple corn beverage "Chicha morada" during dental bleaching. In vitro. Int. J. Odontostomat., 12(4):416-422, 2018.

ABSTRACT: The result of tooth whitening treatment may be affected in patients who consume staining drinks during treatment. The aim of this work was to evaluate the in vitro effect of the exposure to purple corn extract (chicha morada) on human enamel color, during and after the treatment of teeth whitening with $35 \%$ hydrogen peroxide. Forty-eight human teeth were used, divided into groups according to the drink to which it was exposed: Peruvian purple corn extract (MM), green tea $(T)$ and distilled water $(A)$; half of the specimens exposed to each drink were subjected to tooth whitening with $35 \%$ hydrogen peroxide during the first days of exposure to pigmentation, resulting in the following groups: GroupA: Without bleaching + purple corn, 
Group B: Without whitening + green tea, Group C: No whitening + distilled water, Group D: With whitening + purple corn, Group E: With whitening + green tea, Group F: With whitening + distilled water. The color changes were measured with a digital spectrophotometer (VITA Easyshade Advance 4.0, VITA, Germany) before bleaching, during whitening, after whitening and at the end of 36 days of exposure to pigments. According to the $\Delta \mathrm{E}$, the purple corn extract differs significantly with the distilled water $(p<0.05)$. Regarding the degree of luminosity, the purple corn extract with whitening presented the lowest luminosity values $(p<0.05)$. As for the chroma, there were no differences between the groups ( $p>$ 0.05). Exposure to purple corn extract pigments the teeth, during whitening the purple corn extract does not affect the treatment, but if the exposure continues after tooth whitening, the color of the teeth will be affected.

KEY WORDS: Hydrogen peroxide, teeth whitening, staining.

\section{REFERENCIAS BIBLIOGRÁFICAS}

Acuña, E. D.; Delgado-Cotrina, L.; Rumiche, F. A. \& Tay, L. Y. Effect of the purple corn beverage "Chicha Morada" in composite resin during dental bleaching. Scientifica, 2016:2970548, 2016.

Adeyemi, A. A.; Jarad, F. D.; de Josselin de Jong, E.; Pender N. \& Higham, S. M. The evaluation of a novel method comparing quantitative light-induced fluorescence (QLF) with spectrophotometry to assess staining and bleaching of teeth. Clin. Oral Investig., 14(1):19-25, 2010.

Attia, M. L.; Aguiar, F. H; Mathias, P.; Ambrosano, G. M.; Fontes, C. M. \& Liporoni, P. C. The effect of coffee solution on tooth color during home bleaching applications. Am. J. Dent., 22(3):175-9, 2009.

Azer, S. S.; Hague, A. L. \& Johnston, W. M. Effect of bleaching on tooth discolouration from food colourant in vitro. J. Dent., 39 Suppl.3:e52-6, 2011.

Bazzi, J. Z.; Bindo, M. J.; Rached, R. N.; Mazur, R. F.; Vieira, S. \& de Souza, E. M. The effect of at-home bleaching and toothbrushing on removal of coffee and cigarette smoke stains and color stability of enamel. J. Am. Dent. Assoc., 143(5):e1-7, 2012.

Berger, S. B.; Coelho, A. S.; Oliveira, V. A.; Cavalli, V. \& Giannini, M. Enamel susceptibility to red wine staining after $35 \%$ hydrogen peroxide bleaching. J. Appl. Oral Sci., 16(3):201-4, 2008.

Briso, A. L.; Fagundes, T. C.; Gallinari, M. O.; Moreira, J.; de Almeida, L.; Rahal, V.; Gonçalves, R. S. \& Santos, P. D. An in situ study of the influence of staining beverages on color alteration of bleached teeth. Oper. Dent., 41(6):627-33, 2016.

Castillo-Ghiotto, G.; Delgado-Cotrina, L. \& Evangelista-Alva, A. Efectos de la Chicha Morada y café sobre el esmalte dental bovino blanqueado con peróxido de hidrógeno. Rev. Estomatol. Hered., 23(2):63-7, 2013.

Côrtes, G.; Pini, N. P.; Lima, D. A.; Liporoni, P. C.; Munin, E.; Ambrosano, G. M.; Aguiar, F. H. \& Lovadino, J. R. Influence of coffee and red wine on tooth color during and after bleaching. Acta Odontol. Scand., 71(6):1475-80, 2013.

D'Arce, M. B.; Lima, D. A.; Aguiar, F. H.; Bertoldo, C. E.; Ambrosano, G. M. \& Lovadino, J. R. Effectiveness of dental bleaching in depth after using different bleaching agents. J. Clin. Exp. Dent., 5(2):e100-7, 2013.

da Costa e Silva, D.; Tiradentes, S. B. S. P.; Parente, R. C. P. \&
Bandeira, M. F. C. L. Color change using HSB color system of dental resin composites immersed in different common Amazon region beverages. Acta Amazon., 39(4):961-8, 2009.

Dahl, J. E. \& Pallesen, U. Tooth bleaching--a critical review of the biological aspects. Crit. Rev. Oral Biol. Med., 14(4):292-304, 2003.

de Alencar E Silva Leite, M. L.; da Cunha Medeiros E Silva, F. D.; Meireles, S. S.; Duarte, R. M. \& Andrade, A. K. The effect of drinks on color stability and surface roughness of nanocomposites. Eur. J. Dent., 8(3):330-6, 2014.

de Araújo, L. S.; dos Santos, P. H.; Anchieta, R. B.; Catelan, A.; Fraga Briso, A. L.; Fraga Zaze, A. C. \& Sundfeld, R. H. Mineral loss and color change of enamel after bleaching and staining solutions combination. J. Biomed. Opt., 18(10):108004, 2013.

Erdemir, U.; Yildiz, E.; Eren, M. M. \& Ozel, S. Surface hardness of different restorative materials after long-term immersion in sports and energy drinks. Dent. Mater. J., 31(5):729-36, 2012.

Griffiths, C. E.; Bailey, J. R.; Jarad, F. D. \& Youngson, C. C. An investigation into most effective method of treating stained teeth: an in vitro study. J. Dent., 36(1):54-62, 2008.

Karadas, M. \& Seven, N. The effect of different drinks on tooth color after home bleaching. Eur. J. Dent., 8(2):249-53, 2014.

Liporoni, P. C.; Souto, C. M.; Pazinatto, R. B.; Cesar, I. C.; de Rego, M. A.; Mathias, P. \& Cavalli, V. Enamel susceptibility to coffee and red wine staining at different intervals elapsed from bleaching: a photoreflectance spectrophotometry analysis. Photomed. Laser Surg., 28 Suppl. 2:S105-9, 2010.

Matis, B. A.; Wang, G.; Matis, J. I.; Cook, N. B. \& Eckert, G. J. White diet: is it necessary during tooth whitening? Oper. Dent., 40(3): 235-40, 2015.

Passamonti, S.; Vrhovsek, U.; Vanzo, A. \& Mattivi, F. Fast access of some grape pigments to the brain. J. Agric. Food Chem., 53(18):7029-34, 2005.

Pirolo, R.; Mondelli, R. F.; Correr, G. M.; Gonzaga, C. C. \& Furuse, A. Y. Effect of coffee and a cola-based soft drink on the color stability of bleached bovine incisors considering the time elapsed after bleaching. J. Appl. Oral Sci., 22(6):534-40, 2014.

Ramos-Escudero, F.; González-Miret, M. L. \& García-Asuero, A. Effect of various extraction systems on the antioxidant activity kinetic and color of extracts from purple corn. Vitae, 19(1):41-8, 2012.

Rezende, M.; Loguercio, A. D.; Reis, A. \& Kossatz, S. Clinical effects of exposure to coffee during at-home vital bleaching. Oper. Dent., 38(6):E229-36, 2013.

Ruyter, I. E.; Nilner, K. \& Moller, B. Color stability of dental composite resin materials for crown and bridge veneers. Dent. Mater., 3(5):246-51, 1987.

Dirección para correspondencia:

Lidia Yileng Tay

Posgrado en Estomatología de la

Universidad Peruana Cayetano Heredia

Av. Salaverry 2475

San Isidro

Lima

PERÚ

Email: lidia.tay.c@upch.pe

Recibido : 06-07-2018

Aceptado : 31-08-2018 